\title{
LES PÉRITHËCES ET LES ASQUES DU LEPTOSPHAERIA (? SENEGALENSIS SEGRETAIN, BAYLET, DARASSE et CAMAIN
}

\section{Les asques et les ascospores}

\author{
A. PARGUEY-LEDUC*, M. C. JANEX-FAVRE*, S. ANDRIEU**, \\ L. LACOSTE*** et F. TRAORE****
}

RÉSUMÉ. La paroi ascale du Leptosphaeria senegalensis se rattache nettement au type bituniqué; elle comporte, au stade final, un exoascus et un endoascus séparés par un espace clair ; l'endoascus contient des fibrilles, réparties en trois sous-couches, et dont les plus internes sont plissées en accordéon.

Les ascospores ont une paroi complexe comportant, de l'intérieur vers l'extérieur : une endospore, une épispore, plus épaisse autour de la moitié antérieure du corps sporal, une périspore et une ectospore, épaissie en une cupule coiffant l'extrémité postérieure.

La comparaison des ascospores de deux Leptosphaeria "vrais" ( $L$. acuta et $L$. maculans) avec celle du L. senegalensis montre que le rattachement de cette dernière espèce au genre Leptosphaeria n'est pas justifié.

\section{Leptosphaeria (?) senegalensis, Segrétain, Baylet, Darasse et Camain Perithecia and asci. II. Asci and ascospores}

SUMMARY. The ascal wall of Leptosphaeria senegalensis typically belongs to the bitunicate type; in its final state it comprises an exoascus separated from the endoascus by a clear space. The three-layered endoascus is formed of fibrillas whose most internal are accordion-like folded. The ascospores have a complex wall; from the interior to the exterior it comprises: an endospore, an irregularly thickened epispore, a perispore, and an ectospore thickened in a cap at the posterior end of the spore.

The comparison of ascospores of two "real" Leptosphaeria (L. acuta et L. maculans) with those of $L$. senegalensis shows that the latter species must no longer be included in the genus Leptos. phaeria.

* Laboratoire de Cryptogamie, Université Pierre et Marie Curie, 9 Quai Saint-Bernard, F 75005 Paris.

** Unité I.N.S.E.R.M.(U. 42), Domaine du C.E.R.T.I.A., 369, rue Jules Guesde, Flers-Bourg, F 59650.Villeneuve-d'Ascq.

*** Laboratoire de Cryptogamie, Université des Sciences et Techniques, F 59655 Villeneuve-d'Ascq Cedex.

**** Laboratoire de Parasitologie, Faculté de Médecine, 15, rue de l'École-de-Médecine, F 75006 Paris. Accepté le II janvier I982. 
Dans une précédente publication (Parguey-Leduc et coll., 1980), nous avons rapporté nos observations sur l'ontogénie et la structure des périthèces du L. senegalensis. Nous nous proposons, dans le présent travail, de compléter cette étude par celle de ses asques et ascospores, observés en microscopie photonique et en microscopie électronique*.

\section{I - Les asques}

1) Formation des asques : les asques naissent soit à partir de filaments ascogènes dangeardiens (fig. 1, à droite), soit à partir de filaments ascogènes simples; dans ce dernier cas, la cellule terminale se transforme directement en un asque, sans intervention de crochets dangeardiens ( fig. 1, à gauche).

Quel que soit le mode de formation, la cellule pro-ascale et la cellule qui lui a donné naissance sont reliées par un pore, généralement obturé par un bouchon synaptique, bien visible sur les coupes axiales ( fig. 1 et 2). Ce bouchon est constitué, dans chaque cellule, d'une masse externe mamelonnée, très opaque aux électrons, et d'une formation interne délimitée, en coupe, par un liseré clair en forme de M et coiffant les deux extrémités pointues des lames septales.

2) Asques jeunes : la cellule pro-ascale, plus ou moins globuleuse, s'allonge très rapidement et se transforme en un jeune asque ( $f$ ig. 1 et 3), entouré par une paroi mince et homogène. Sa partie supérieure est occupée par le réticulum endoplasmique (fig. 3), alors que sa partie inférieure renferme de nombreuses petites vacuoles; certaines d'entre elles contiennent un globule très opaque aux électrons, correspondant probablement aux précipités métachromatiques visibles en microscopie photonique. Entre ces deux parties est logé le volumineux noyau diploïde, contenant un nucléole très chromophile et plusieurs masses chromatiques. Quelques globules lipidiques, répartis de façon désordonnée dans l'asque, sont également visibles.

3) Asques adultes : portés par un pied aminci ou non et en forme de massue plus ou moins allongée (fig. 4 et 5), les asques adultes ont de 60 à $100 \mu \mathrm{m} \times 12$ à $20 \mu \mathrm{m}$.

Ils contiennent huit ascospores phragmiées, incolores, entourées par une paroi inégalement épaissie. Au sommet de l'asque l'épiplasme se rétrécit brusquement dans une chambre apicale courte et cylindrique, dans laquelle n'a été observé aucun appareil apical.

La déhiscence de l'asque se produit par le mécanisme du " Jack-in-box » (fig. 5), caractéristique des Bituniqués.

4) Évolution de la paroi ascale: celle-ci est du type bituniqué. Son évolution et sa structure ont été suivies au microscope électronique. Elles rappellent celles que nous avons déjà décrites chez divers autres Bituniqués (Parguey-Leduc et JanexFavre, 1982).

* Nous avons plaisir à remercier N. JAmpsin et C. Fournigault pour leur précieuse collaboration technique. 

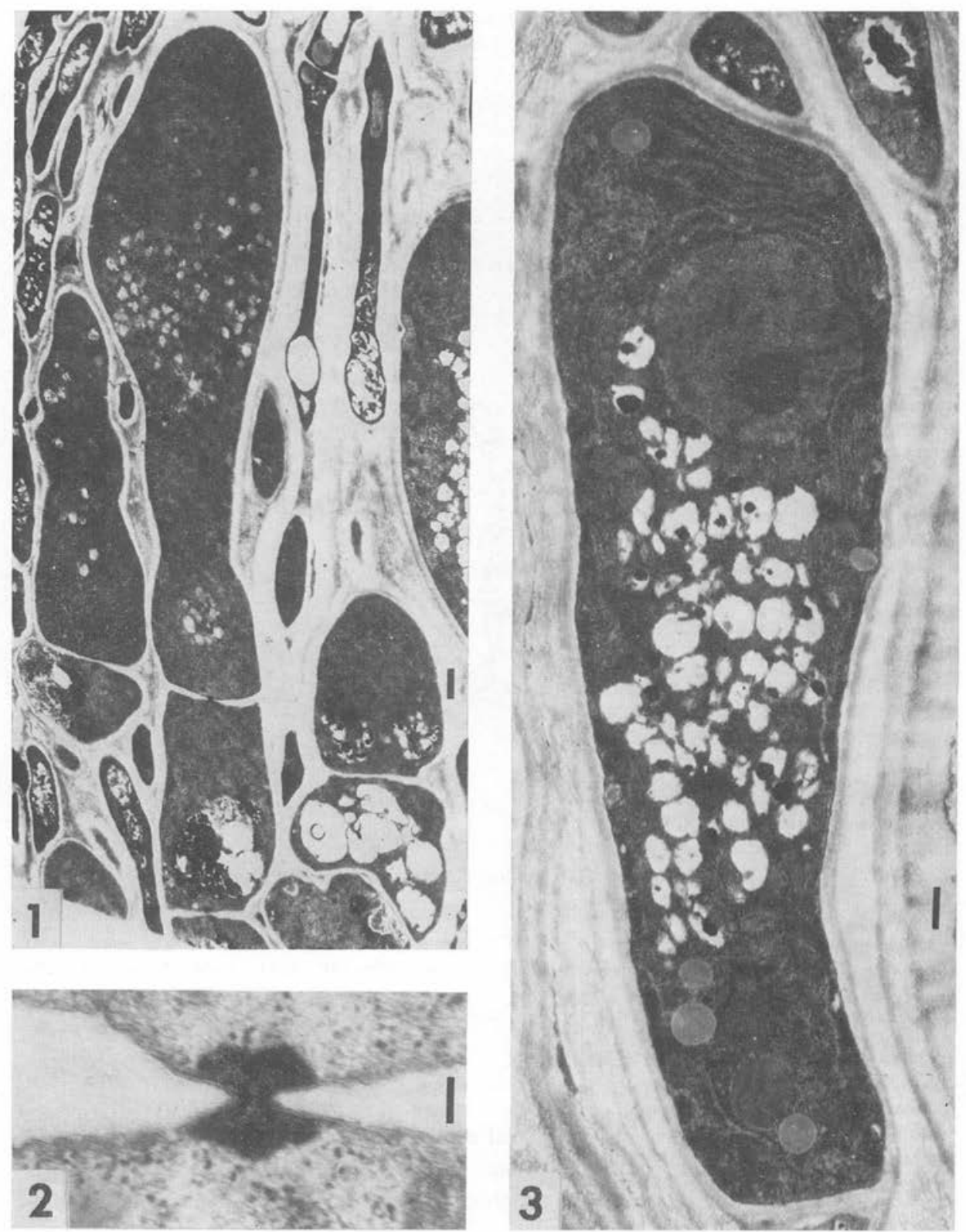

FIG. I-3. - Jeunes asques, microscopie électronique.

Fig. I : formation des asques, à partir de filaments ascogènes dangeardiens (à droite) ou simples (à gauche).

Fig. 2: détail du bouchon synaptique entre la cellule pro-ascale et celle qui lui a donné naissance.

Fig. 3 : jeune asque avec répartition superposée de ses constituants.

Échelle: I $\mu \mathrm{m}$ (fig. I) ; o, I $\mu \mathrm{m}$ (fig. 2) ; 0,5 $\mu \mathrm{m}$ (fig. 3). 


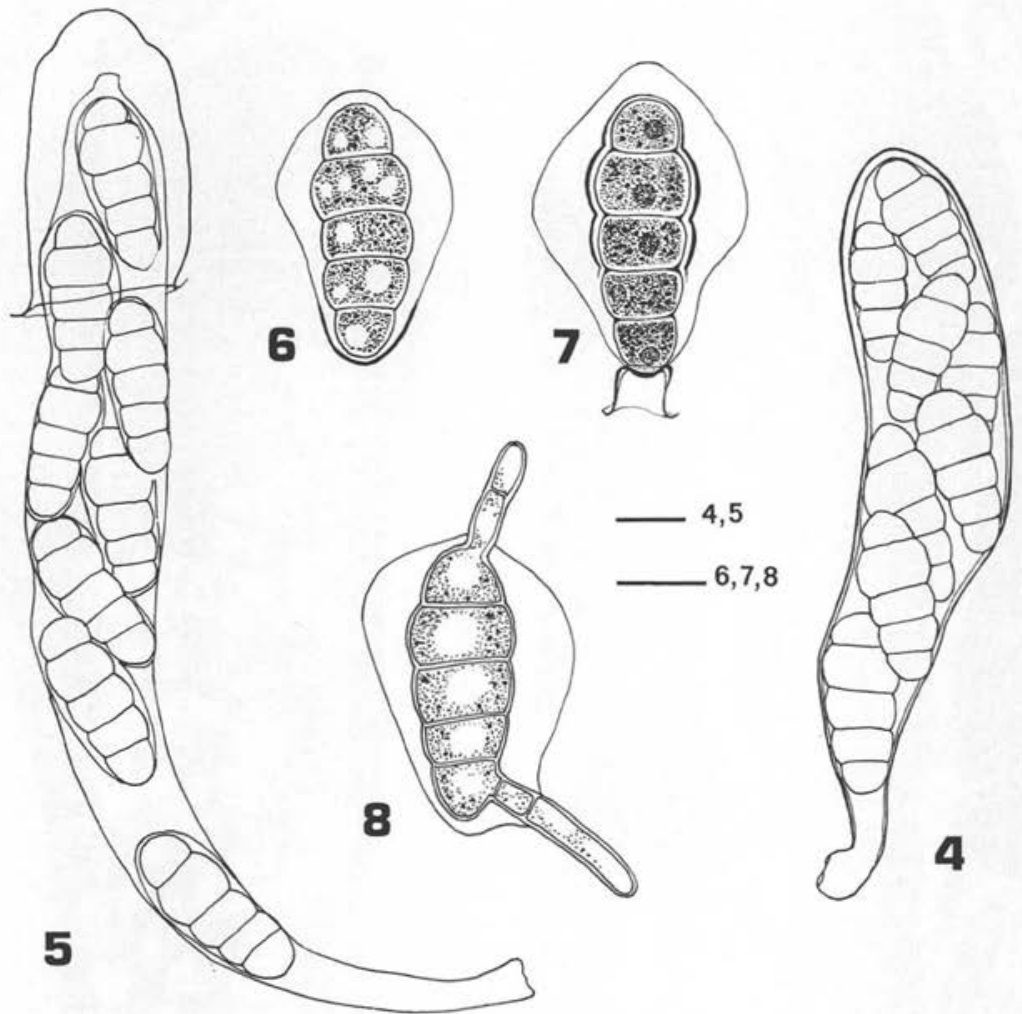

FIG. 4-8. - Asques et ascospores adultes, microscopie photonique.

Fig. 4: asque adulte (montage dans l'eau).

Fig. S: déhiscence de l'asque par Jack-in-box (montage dans l'eau).

Fig. 6 et $7:$ ascospores adultes (coloration par le vert Visba (fig. 6) et l'encre Waterman bleue (fig. 7)).

Fig. 8: ascospore en germination (coloration par le bleu Coton).

Echelle : io $\mu \mathrm{m}$.

Les asques très jeunes ont une paroi mince, homogène, finement granuleuse et porteuse, vers l'extérieur, d'une substance interascale réticulée (fig. 9).

Ensuite, la paroi se subdivise progressivement en deux couches : une couche interne réticulo-granuleuse, moyennement dense aux électrons, et une couche externe de même texture, mais plus sombre, et toujours en continuité avec la substance interascale (fig. 10).

Plus tard, entre ces deux couches, se forme l'espace clair séparant l'exoascus et l'endoascus (flèche, fig. 11), que nous avons déjà décrit chez cette espèce comme chez plusieurs autres Bituniqués (Parguey-Leduc et Janex-Favre, 1982). 

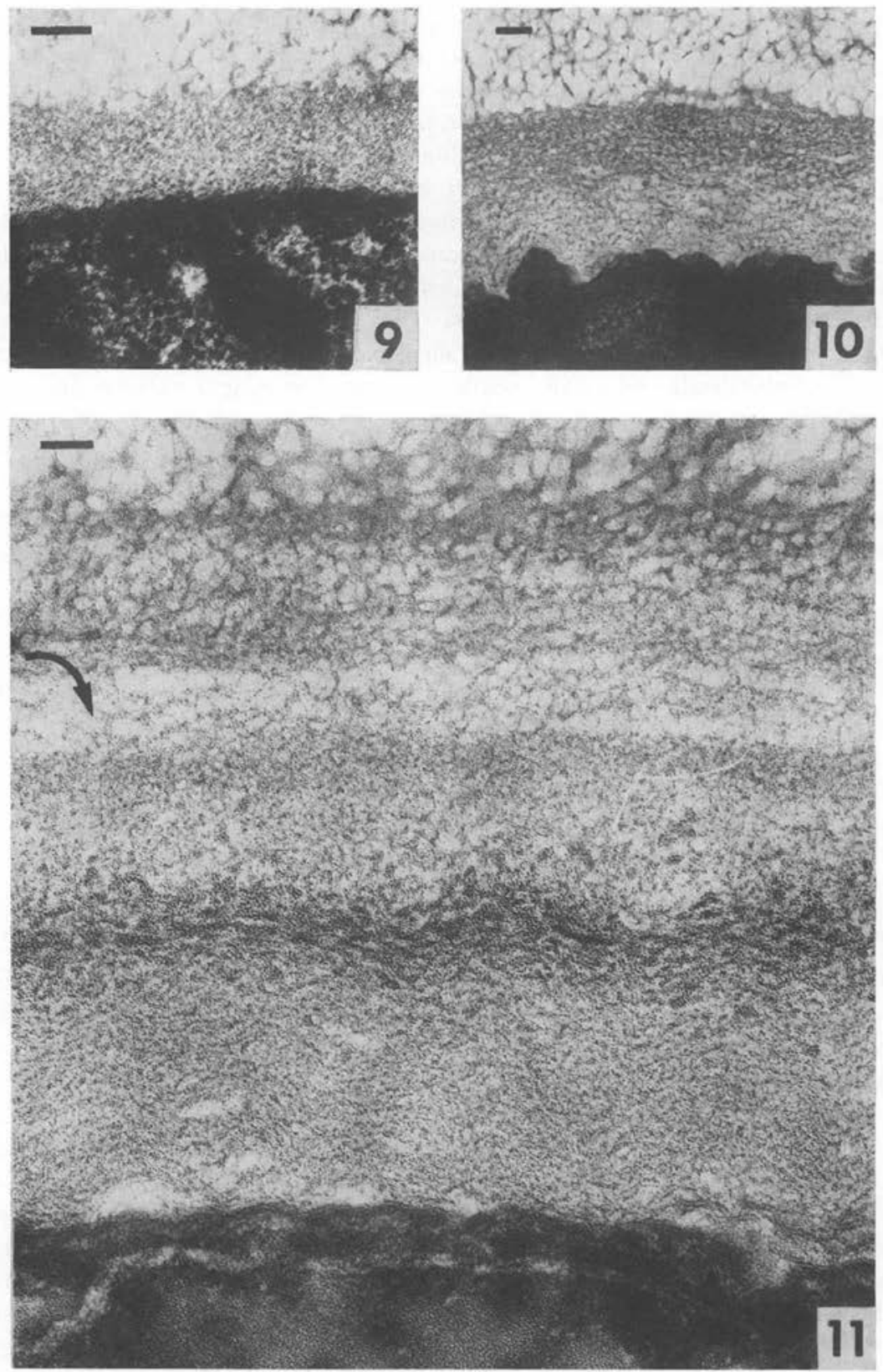

FIG. 9-II. - Évolution de la paroi ascale, microscopie électronique (la face externe de l'asque est située vers le haut).

Fig. 9: paroi homogène.

Fig. 10: paroi subdivisée en deux couches.

Fig. II : formation de l'espace clair séparant l'exoascus et l'endoascus (flèche). Echelle : o, $1 \mu \mathrm{m}$. 
L'exoascus demeure inchangé, tandis que l'endoascus se complique, arec la délimitation de trois sous-couches. En effet, une sous-couche moyenne sombre devient distincte, dans laquelle les granules s'ordonnent en fibrilles très opaques aux électrons ; ces fibrilles tendent à s'empiler et à devenir onduleuses ; ce phénomène se poursuit ensuite de part et d'autre dans les sous-couches interne et externe de l'endoascus (fig. 12). Ensuite, dans la sous-couche interne, les fibrilles se plissent très fortement en accordéon, dessinant des zig-zags aigus, qui suivent fidèlement les indentations de plasmalemme ( $f$ ig. 13).

Puis l'exoascus se divise à son tour en deux sous-couches (fig. 12 et 13) : une sous-couche superficielle très fine, sombre, et une sous-couche interne plus épaisse et plus claire.

Ainsi, à l'état adulte, la paroi ascale du L. senegalensis est très complexe, comprenant 5 sous-couches, 2 pour l'exoascus et 3 pour l'endoascus. Une aussi grande complexité de l'endoascus n'a pas été rencontrée chez les espèces précédemment examinées (Parguey-Leduc et Janex-Favre, 1982) où l'endoascus est, en règle générale, constitué par une couche unique, à fibrilles toujours disposées en zig-zags, comme celles de la sous-couche interne de l'endoascus, chez le Leptosphaeria senegalensis.

Malgré sa complexité, la paroi de ce Pyrénomycète présente les caractéristiques essentielles du type bituniqué, à savoir :

1. La présence d'un espace clair entre l'exo- et l'endoascus ;

2. La disposition en accordéon des fibrilles de l'endoascus interne.

\section{II - Les ascospores}

\section{1) Morphologie, en microscopie photonique}

Oblongues et incolores, de 12-18 × 4-7 $\mu \mathrm{m}$, les ascospores adultes du L. senegalensis sont cloisonnées en cinq cellules de taille inégale, séparées les unes des autres par des constrictions bien marquées ( fig. 6) ; c'est généralement la cellule sub-apicale qui est la plus grosse et la plus renflée.

Lorsque les ascospores sont montées dans un milieu aqueux (encre Waterman bleue, vert Visba) et émises hors de l'asque, elles apparaissent constamment, en microscopie photonique, entourées par une paroi fortement dilatée. Cette dilatation est irrégulière : elle atteint son plus grand diamètre autour de la moitié antérieure de l'ascospore, tandis qu'elle est quasiment nulle à son pôle postérieur. Celui-ci, par contre, est coiffé par une cupule épaissie, colorable en bleu par l'encre et le vert Visba. Dans les ascospores parfaitement mûres et lorsque l'imbibition est maximale, cette cupule se désolidarise du reste de la paroi et se retourne en doigt de gant (fig. 7). Cette formation correspond à ce que El Ani et Gordon (1965) dénomment " basal satellite ». La coloration à l'encre met également en évidence d'une part une lame médiane dans les septums, séparant les cellules successives d'une ascospore, et d'autre part, sous la couche dilatée de la paroi ascosporale, un manchon limité à la moitié antérieure de l'ascospore. 

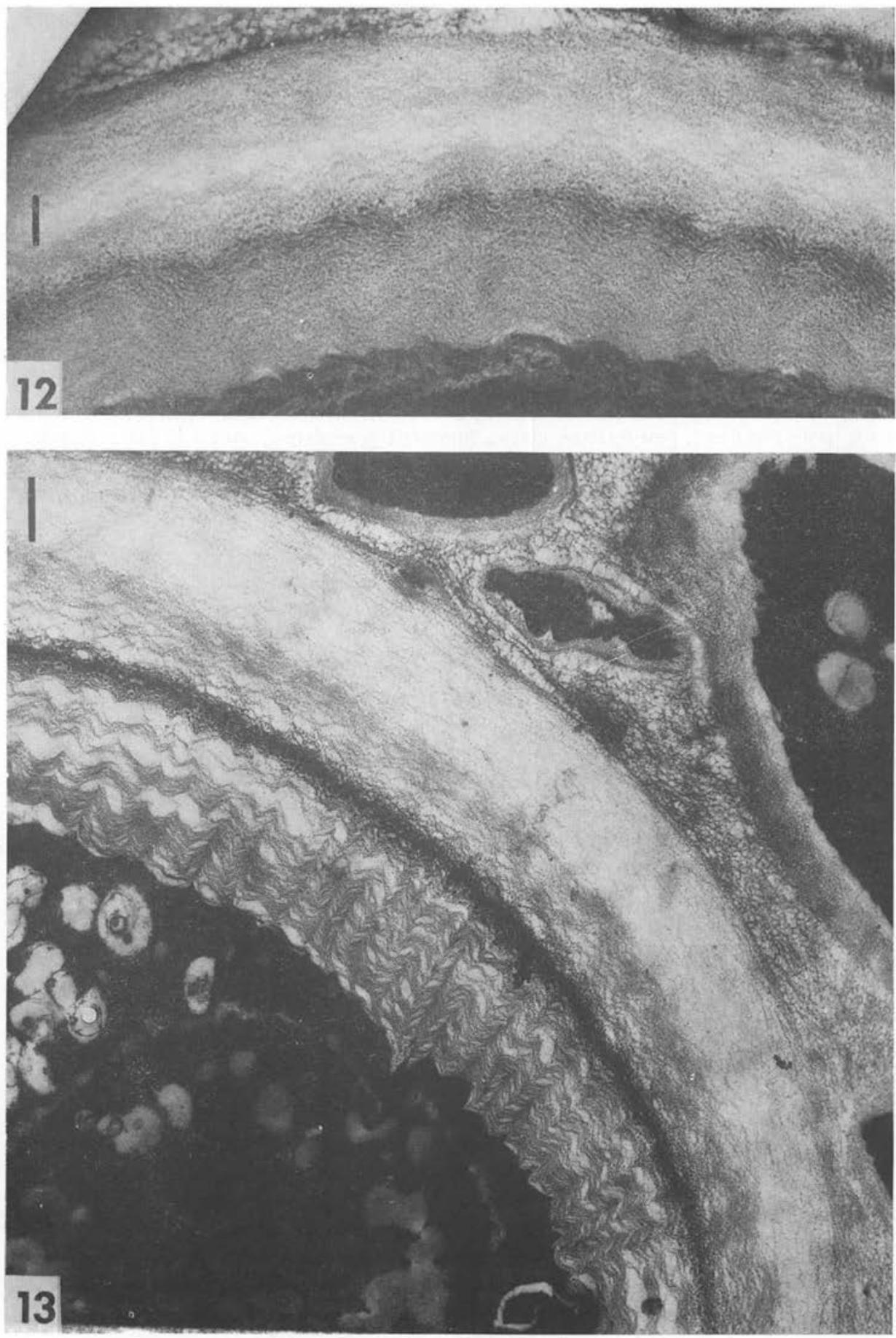

FIG. I2-13. - Évolution de la paroi ascale, microscopie électronique (la face externe de l'asque est située vers le haut).

Structure de thexoascus en $2^{\prime}$ sous-couches et arrangement des fibrilles de l'endoascus en 3 souscouches, dans lesquelles celles-ci diffèrent par leur densité et leur degré d'ondulation.

Échelle : o,2 $\mu \mathrm{m}$ (fig. 12) et $0,5 \mu \mathrm{m}$ (fig. 13). 
Les ascospores germent facilement dans l'eau. En règle générale, ce sont les deux cellules terminales qui produisent des tubes germinatifs ( fig. 8); exceptionnellement, une cellule intermédiaire peut également émettre un tel tube.

\section{2) Structure et évolution de la paroi, en microscopie électronique}

Les plus jeunes ascospores observées (fig. 14 et $21 \mathrm{~A}$ ) ont une paroi sinueuse limitée sur ses deux faces par une membrane tripartite (flèches), dont l'interne constitue le plasmalemme sporal. Accolée à chacune des membranes se trouve une zone granuleuse, moyennement opaque aux électrons. Entre ces deux membranes, on observe une bande très claire.

A un stade ultérieur ( $f i g .15$ et $21 \mathrm{~B}$ ), entre le plasmalemme et la membrane limitante externe, toujours nettement tripartite, se différencient deux couches : une endospore régulière, peu épaisse, claire, finement granuleuse, contre le plasmalemme, et une épispore irrégulière, épaisse, à texture hétérogène. Sa limite interne est très précocement précisée sous forme d'une ligne mince appliquée contre l'endospore. Cette ligne est porteuse, vers l'extérieur, de pointes (fig. 15 et 21 B) qui, en relation avec des masses opaques aux électrons situées au sein de l'épispore (fig. 16 et $21 \mathrm{C}$ ) forment finalement des piliers épisporiques rattachés vers le haut à la partie externe, finement granuleuse, de l'épispore. Entre les piliers sont répartis des faisceaux de mèches désordonnées ( fig. 15 et 21 B).

Ensuite se différencie, entre l'épispore et la membrane limitante externe, toujours tripartite, la périspore, peu épaisse, granuleuse et moyennement dense aux électrons (fig. 16 et 21 C).

Au stade final, la paroi ascosporale est encore plus complexe puisqu'aux couches déjà formées s'ajoute une ectospore dérivée de la membrane limitante externe. De plus, l'épaisseur de deux des couches varie suivant le niveau considéré. En effet, sur une coupe longitudinale ( fig. 17), il apparaît que l'épispore est fortement dilatée au niveau de la cellule sub-apicale renflée tandis que l'ectospore s'épaissit progressivement dans la moitié postérieure de l'ascospore (fig. 18).

Cela est particulièrement frappant si l'on compare deux coupes transversales d'ascospores adultes effectuées l'une dans la cellule antérieure ( $f i g .19$ et 21 D) et l'autre dans la cellule postérieure ( fig. 20 et $21 \mathrm{D}^{\prime}$ ). Dans la première c'est l'épispore qui est la plus importante, avec ses piliers et mèches, tandis que dans la seconde c'est l'ectospore sombre aux électrons et d'aspect moucheté.

\section{3) Conclusion}

Ces observations ultrastructurales permettent d'interpréter les données obtenues en microscopie photonique. Ainsi, la paroi des ascospores du L. senegalensis comprend, de l'intérieur vers l'extérieur:

1. l'endospore qui entoure individuellement chacune des cellules ;

2. l'épispore, plus développée dans la partie antérieure de la spore ( fig. 7 et 17) : visible en microscopie photonique sous forme d'un manchon colorable en bleu par l'encre ( $f i g .7$ ), elle présente sur les coupes ultra-fines une texture complexe et originale ( $f$ ig. 16): 

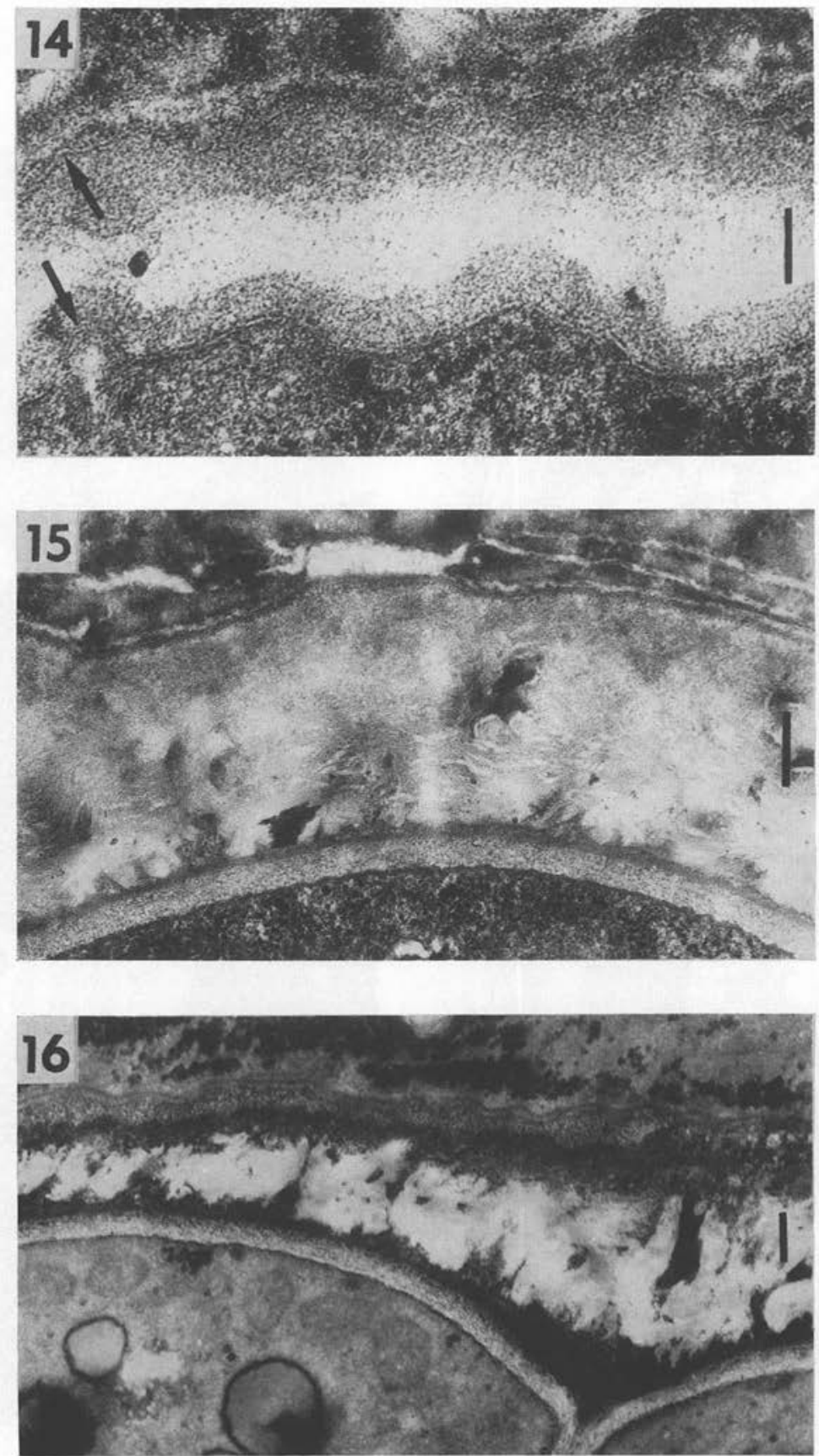

FIG. I4-I6. - Évolution de la paroi des ascospores, microscopie électronique (la face externe des ascopores est située vers le haut).

Fig. 14 : paroi d'une très jeune ascospore.

Fig. Is : différenciation de l'endo- et de l'épispore.

Fig. 16 : formation des piliers épisporiques.

Echelle : $0,2 \mu \mathrm{m}$. 

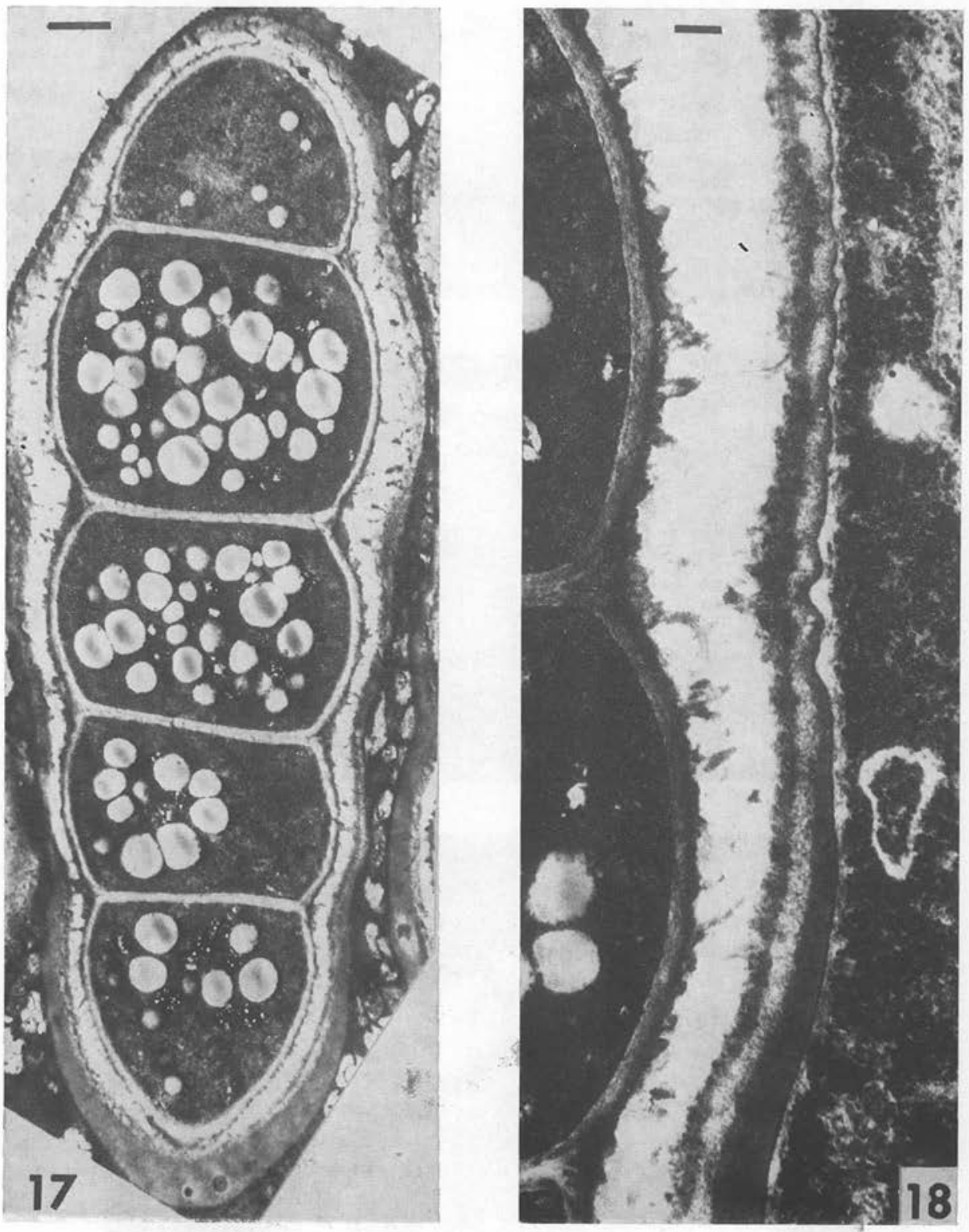

FIG. 17-18. - Paroi des ascospores adultes, microscopie électronique.

Fig. 17 : stade final.

Fig. I8: détail de la partie moyenne d'une ascospore adulte montrant l'épaississement progressif de l'ectospore.

Échelle: $\mathrm{I} \mu \mathrm{m}$ (fig. $\mathrm{I} 7$ ) et $0,25 \mu \mathrm{m}$ (fig. $\mathrm{r} 8$ ). 

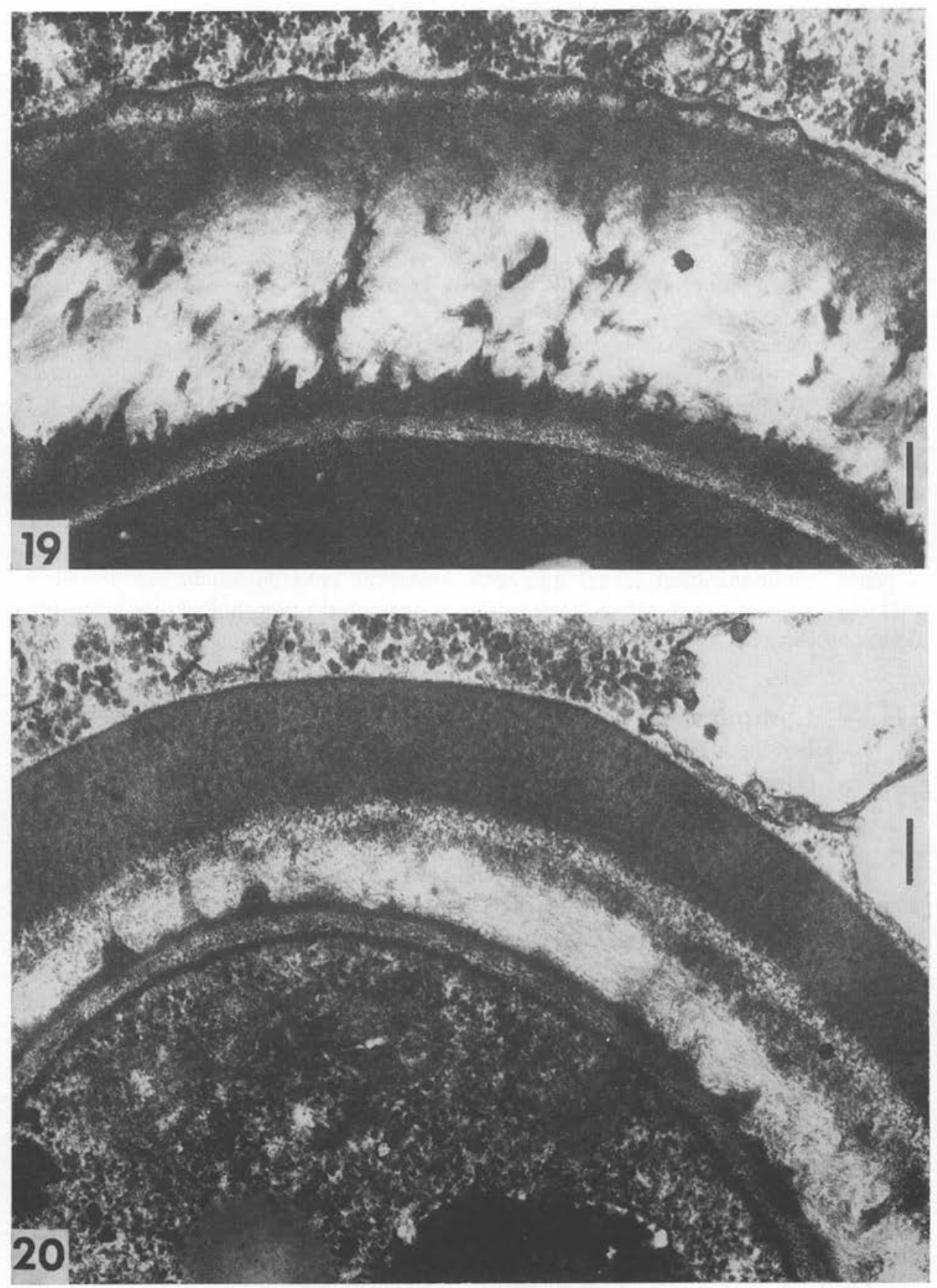

Fig. 19-20. - Paroi des ascospores adultes, microscopie électronique. Coupes transversales passant par la cellule antérieure (fig. 19) et par la cellule postérieure (fig. 20).

Échelle : $0,2 \mu \mathrm{m}$. 
3. la périspore : selon la règle, elle se dilate fortement, en milieu aqueux, autour des ascospores émises hors de l'asque ( fig. 6 et 7); elle apparaît au contraire mince et régulière sur les coupes ultra-fines d'ascospores demeurées dans l'asque ( fig. 16 et 18);

4. l'ectospore : en microscopie photonique, elle souligne la limite externe de la périspore dilatée et s'épaissit à la partie postérieure de l'ascospore en formant une cupule ( $f$ ig. 6 et 7 ) : celle-ci est également bien visible en microscopie électronique, où il apparaît clairement qu'elle résulte de l'épaississement progressif de l'ectospore ( $f$ ig. 18).

La complexité de cette paroi n'avait pas jusqu'ici été reconnue. Toutefois, El Ani et Gordon (1965) ont observé la présence constante d'une couche fortement dilatable autour de l'ascospore du L. senegalensis, caractère qui n'avait pas été signalé par Segretain et ses collaborateurs (1959) lors de leur description de l'espèce. Pour sa part, Lacoste avait, en 1965, noté la présence d'une " gaine muqueuse " autour des ascospores, et cela avait fait douter cet auteur de l'appartenance du L. senegalensis au genre Leptosphaeria, les autres espèces ne présentant jamais ce caractère. Quant à Holm, à qui des échantillons du L. senegalensis ont été soumis, il pense (communication écrite) que cette espèce ne peut en aucun cas demeurer rattachée au genre Leptosphaeria, en raison des caractères morphologiques originaux de ses ascospores.

\section{III - Comparaison des ascospores du Leptosphaeria senega- lensis à celles de deux Leptosphaeria « vrais »: le L. acuta (Moug. et Nestl.), Karten et le L. maculans (Desm.) Ces. et de Not.}

En observation photonique, les différences les plus marquantes entre les ascospores du L. senegalensis et celles des deux Leptosphaeria vrais sont :

1. Les ascospores du L. senegalensis sont notablement plus petites que celles des deux autres espèces ;

2. Elles demeurent hyalines, alors que celles des Leptosphaeria vrais sont plus ou moins pigmentées ;

3. Elles présentent au niveau des cloisons intercellulaires de fortes constrictions, qui sont absentes chez les deux autres espèces ;

4. La forte dilatation de la périspore, en milieu aqueux, n'existe que chez le $L$. senegalensis.

L'étude ultrastructurale fait apparaître chez ces trois espèces de Leptosphaeria d'autres différences concernant d'une part la structure de la paroi ascosporale et d'autre part celle des septums intercellulaires.

La paroi ascosporale est beaucoup moins épaisse chez les Leptosphaeria vrais que chez le $L$. senegalensis. Chez les trois espèces l'endospore entoure classiquement chaque cellule, mais chez le $L$. senegalensis elle s'incurve progressivement vers 
21

A

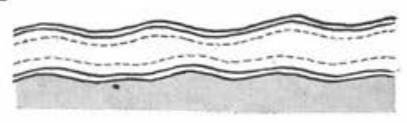

B
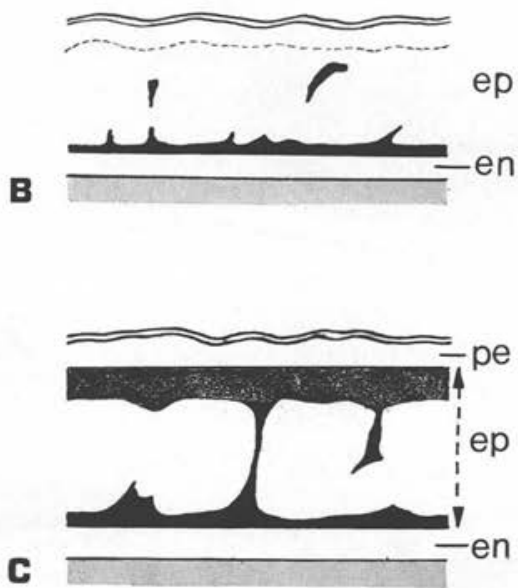

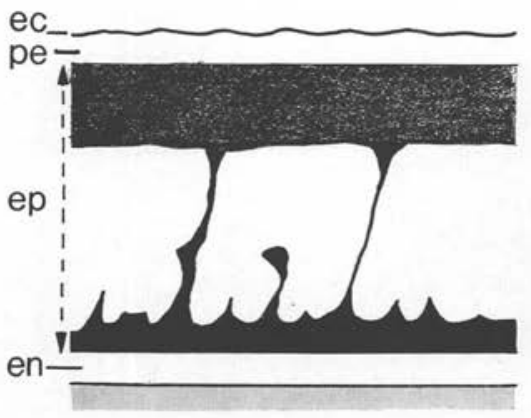

D

Fig. 21. - Schémas montrant l'évolution de la paroi ascosporale (de A à C) et son état final (D : au niveau de la cellule antérieure, D' au niveau de la cellule postérieure). $e c$ : ectospore ; $e n$ : endospore ; ep : epispore, pe : périspore.

l'intérieur au niveau des septums intercellulaires, ce qui détermine les constrictions caractéristiques de cette espèce; chaque cellule sporale a la forme d'un tonnelet (fig. 17, 18, 24 et 27 A). Au contraire, chez le L. acuta (fig. 22, 25 et 27 B) et le L. maculans ( fig. 23, 26 et 27 C) l'endospore demeure rectiligne latéralement et s'incurve brutalement à angle droit au niveau des septums; les cellules présentent alors une forme cylindrique.

L'épispore, particulièrement bien développée chez le $L$. senegalensis est également présente chez le $L$. maculans, sous forme d'une mince couche fibrilleuse ; elle n'est pas distincte chez le $L$. acuta.

L'exospore, rectiligne et sombre, est bien caractérisée chez les deux Leptosphaeria vrais. Par contre, elle manque chez le $L$. senegalensis, ce qui pourrait expliquer que les ascospores de cette espèce demeurent claires, contrairement à celles des deux autres espèces.

La périspore et l'ectospore présentent également des caractères différents selon les espèces. Alors que chez le $L$. senegalensis, ainsi qu'on l'a dit plus haut, l'ectospore, régulièrement appliquée contre la périspore, s'épaissit en une cupule à la partie postérieure de l'ascospore, elle demeure très fine chez le $L$. acuta et le $L$. maculans. Chez ces deux espèces, elle est sinueuse et laisse apparaitre une périspore claire au niveau de ses plus amples sinuosités. 

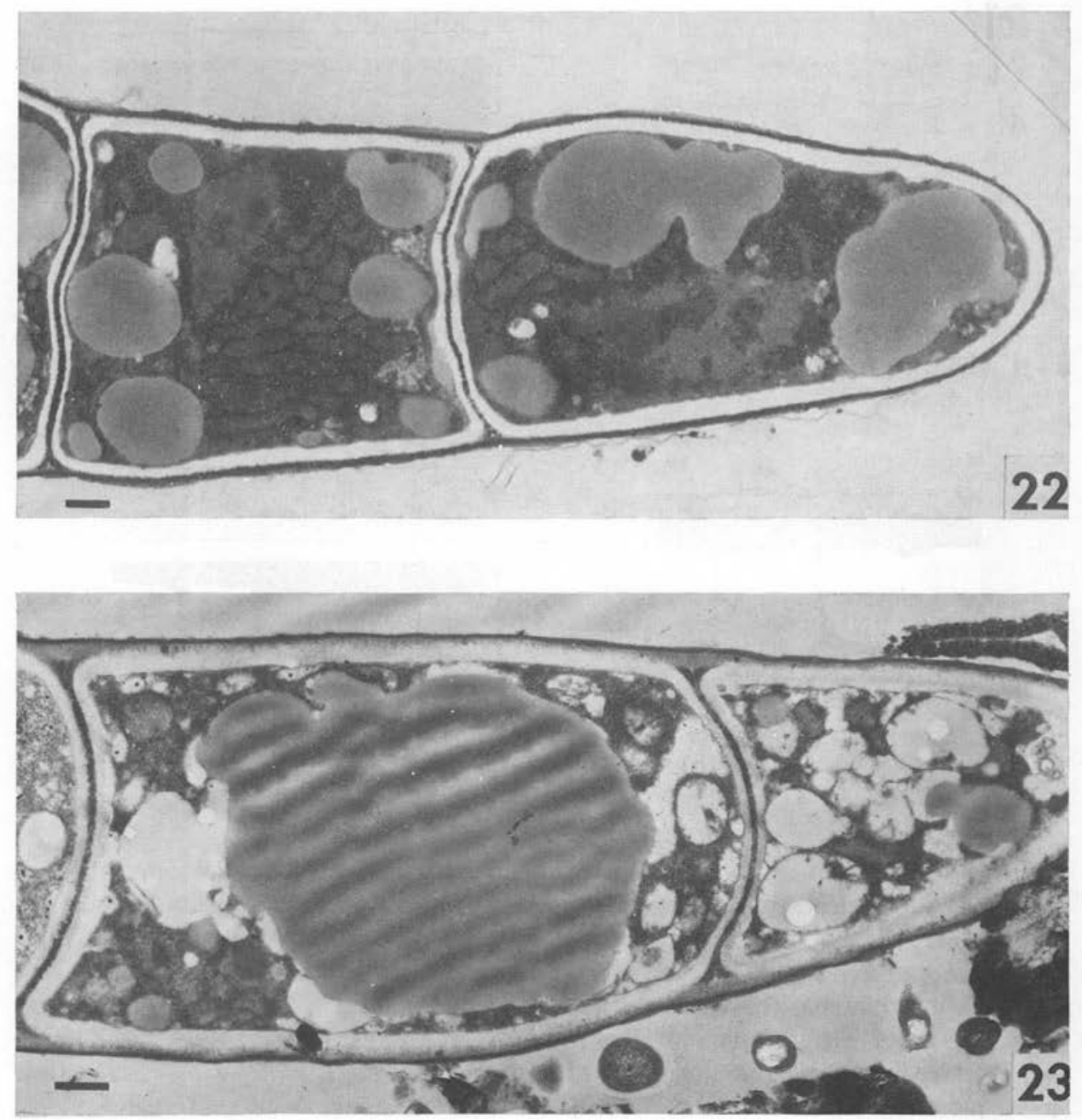

FIG. 22-23. - Parties terminales d'ascospores du L. acuta (fig. 22) et du L. maculans (fig. 23); microscopie électronique.

Échelle: $0,5 \mu \mathrm{m}$.

En ce qui concerne les septums intercellulaires, des différences notables existent également entre les espèces examinées. Chez le $L$. senegalensis, ils sont pratiquement constitués uniquement par les endospores accolées des deux cellules contiguës, parfois séparées par un mince espace clair en forme de lame ; ils présentent un renflement autour du pore septal central. Chez le L. acuta (fig. 25 et 27 B) et le L. maculans ( fig. 26 et $27 \mathrm{C}$ ), une formation sombre aux électrons, parcourue en son centre par 

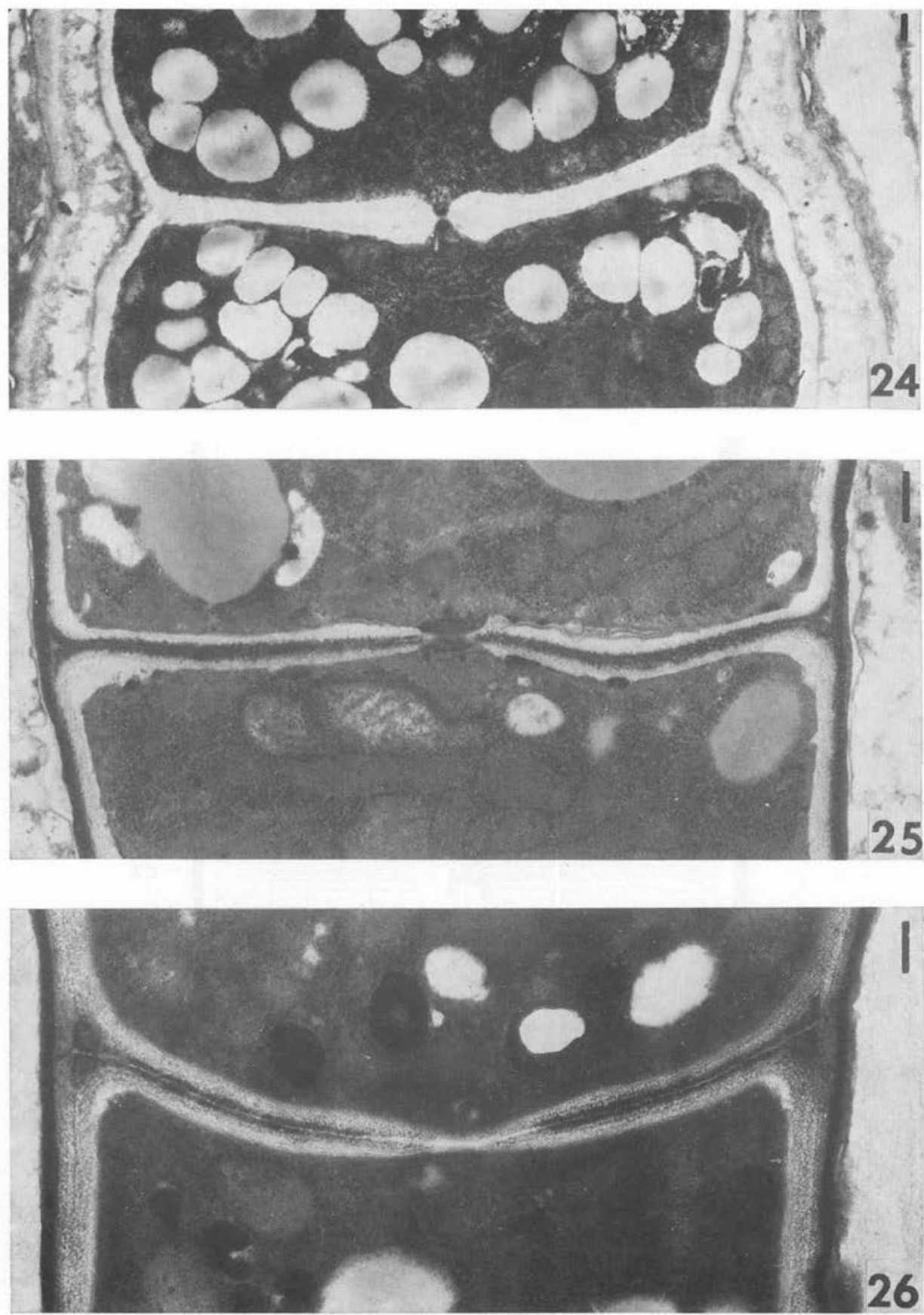

FIG. 24-26. - Septums du L. senegalensis (fig. 24), du L. acuta (fig. 25) et du L. maculans (fig. 26); microscopie électronique.

Echelle: $0,2 \mu \mathrm{m}$. 

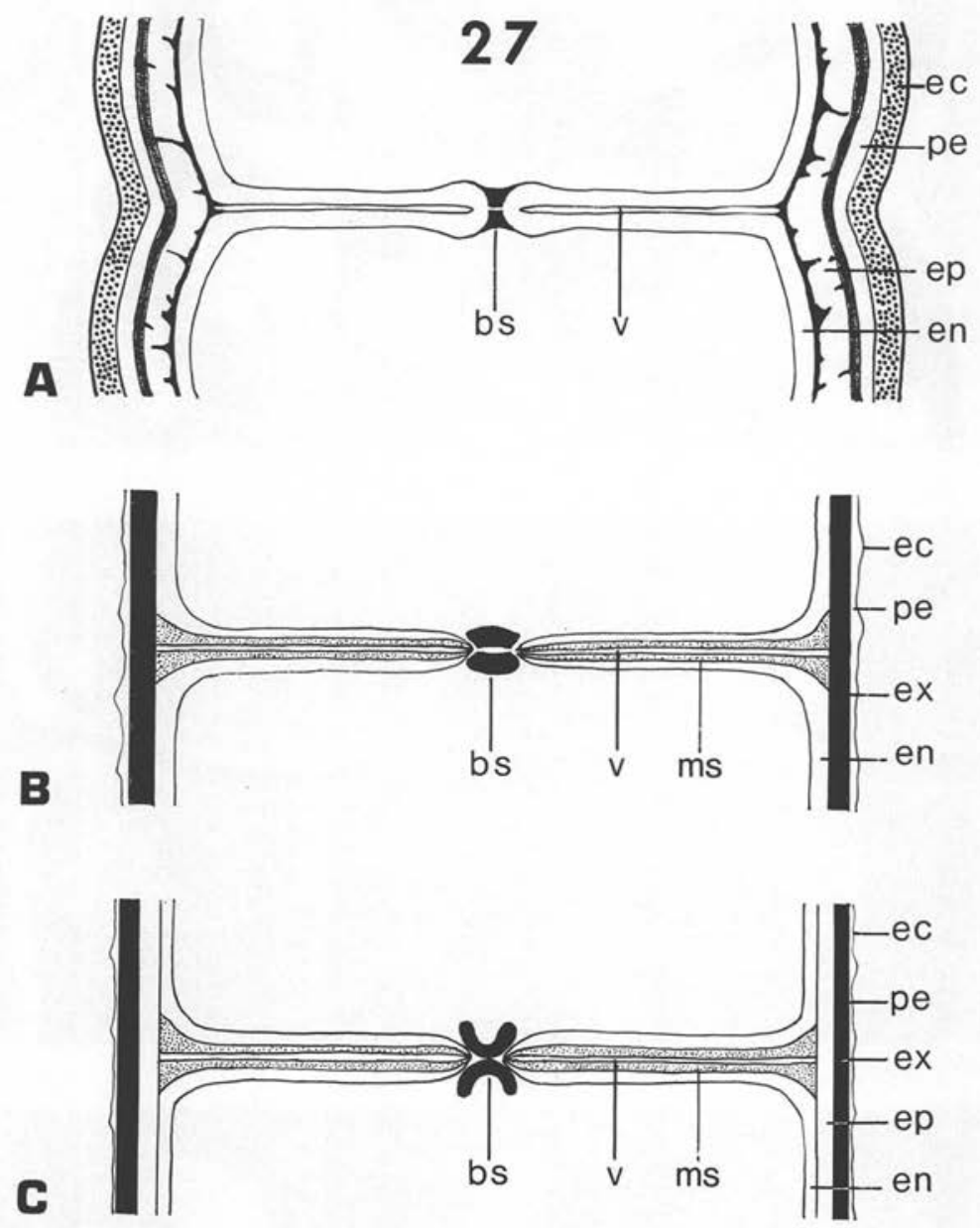

FIG. 27. - Schémas comparatifs des septums du L. senegalensis (A), du $L$. acuta (B) et du $L$. maculans (C).

$b s$ : bouchon synaptique ; ec : ectospore ; en : endospore ; ep : épispore ; ex : exospore ; $m s$ : mésospore; pe : périspore; $v$ : espace clair médian du septum intercellulaire.

un espace clair, s'intercale entre les endospores; on peut l'interpréter comme une mésospore. Cette formation s'amincit progressivement en pointe au niveau du pore septal.

Les pores septaux sont visibles chez les trois espèces sous forme de deux bouchons accolés, denses aux électrons, de forme et de taille différentes selon les espèces, ceux de $L$. senegalensis étant les plus petits. 
Les multiples différences apparues, tant en microscopie photonique qu'en microscopie électronique, entre les ascospores du L. senegalensis et celles de deux Leptosphaeria vrais, indiquent clairement que le $L$. senegalensis ne peut en aucune manière être rangé parmi les Leptosphaeria vrais. Il est donc indispensable de reconsidérer la position systématique de ce Pyrénomycète et sa dénomination.

\section{Note}

Technique utilisée pour l'étude en microscopie électronique : fixation par le glutaraldéhyde et le tétroxyde d'osmium, contraste par l'acétate d'uranyle et le citrate de plomb.

\section{BIBLIOGRAPHIE}

Chadefaud M. : Une interprétation de la paroi des ascospores septées, notamment celles des Aglaospora et des Pleospora. Bull. Soc. Myc. Fr., 1969, 85, 145-1 58.

EL ANI A. S., GoRDoN M. A. : The ascospore sheath and taxonomy of Leptosphaeria senegalensis. Mycologia, 1965, $57,275-278$.

LAcoste L. : Biologie naturelle et culturale du genre Leptosphaeria Cesati et de Notaris. Déterminisme de la reproduction sexuelle. Thèse de Doctorat ès Sciences, 1965, Toulouse.

Parguey-Leduc A., ANDrieu S., Traore F., Lacoste L. : Les périthèces et les asques du Leptosphaeria (?) senegalensis Segretain, Baylet, Darasse et Camain. I. L'ontogénie et la structure des périthèces. Ann. Parasitol. Hum. Comp., 1981, s6, 225-236.

PARguey-Leduc A., Janex-FAvre M. C. : La paroi des asques chez les Pyrénomycètes : étude ultrastructurale. I. Les asques bituniqués typiques. Can. J. Bot,, I982 (à paraître).

Segretain G., Baylet J., Darasse H., Camain R. : Leptosphaeria senegalensis n. sp., agent du mycétome à grains noirs. C. R. Acad. Sci. Paris, 1959, 3730-3732. 